\title{
Treatment of the wide open wound in the Ehlers- Danlos syndrome
}

\author{
Bong Soo Baik, \\ Wu Seop Lee, \\ Ki Sung Park, \\ Wan Suk Yang, \\ So Young Ji \\ Department of Plastic and \\ Reconstructive Surgery, Dong-Kang \\ General Hospital, Ulsan, Korea
}

Ehlers-Danlos syndrome (EDS) is an inherited disorder of collagen biosynthesis and structure, characterized by skin hyperextensibility, joint hypermobility, aberrant scars, and tissue friability. Besides the skin, skeleton (joint) and vessels, other organs such as the eyes and the intestine can be affected in this syndrome. Accordingly, interdisciplinary cooperation is necessary for a successful treatment. Three basic surgical problems are arising due to an EDS: decreased the strength of the tissue causes making the wound dehiscence, increased bleeding tendency due to the blood vessel fragility, and delayed wound healing period. Surgery patients with an EDS require an experienced surgeon in treating EDS patients; the treatment process requires careful tissue handling and a long postoperative care. A surgeon should also recognize whether the patient shows a resistance to local anesthetics and a high risk of hematoma formation. This report describes a patient with a wide open wound on the foot dorsum and delayed wound healing after the primary approximation of the wound margins.

Keywords: Connective tissue diseases / Ehlers-Danlos syndrome / Joint instability

\section{INTRODUCTION}

The Ehlers-Danlos syndrome (EDS) is a group of hereditary disorders, and its central pathogenesis is the genetic defects of the collagen. Six main types of EDS are classical, hypermobile, vascular, kyphoscoliotic $\mathrm{A} / \mathrm{B}$, arthrochalasis $\mathrm{A} / \mathrm{B}$, dermatosparaxis types $[1,2]$. Careful clinical and morphological examinations are prerequisite for diagnosing EDS. Cardinal symptoms affect the skin and the skeletal system. The skin is velvety, soft and is typically hyperelastic and hyperextensible. The skin is fragile and tends to bruise easily even after a minor trauma. It tends to form atrophic, hyper and hypopigmented scars. Hypermobility may affect the small and large joints and may lead to joint dislocation, effusion, as well as arthritis. To confirm the

Correspondence: So Young Ji

Department of Plastic and Reconstructive Surgery, Dong-Kang General Hospital, 239 Taehwa-ro, Jung-gu, Ulsan 44455, Korea

E-mail: csy0203@hanmail.net

Received November 12, 2018 / Revised January 15, 2019 / Accepted January 16, 2019 diagnosis, deep biopsy, containing the entire dermis, is necessary and electron microscopy should always be performed.

\section{CASE REPORT}

A 41-year-old Australian man was presented to Department of Plastic and Reconstructive Surgery, Dong-Kang General Hospital, with an open wound on the right lateral foot between lateral malleolus and plantar area of the heel, and the following injury was caused by slipping down on the wet floor about a month previously.

The patient has been diagnosed with a mild form of EDS and experienced wide scarring on the bony protrusion areas, especially on the anterior portion of the knees, a trauma left from his childhood (Fig. 1A). The physical examination revealed the skin hyperextensibility on the neck (Fig. 1B), but the hand joint was not overextensible. His toes were laterally deviated (equinovalgus) with medial protrusion of the metatarsophalangeal joint, 


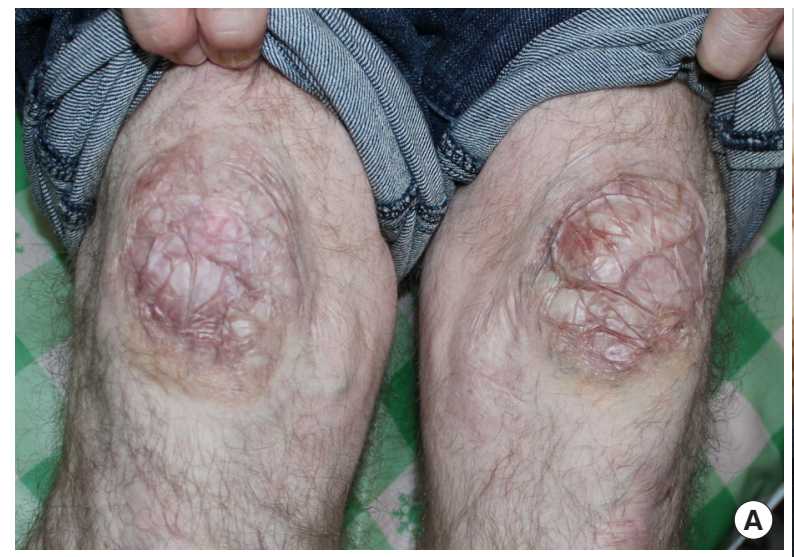

Fig. 1. Physical findings. (A) Wide scars on the anterior knees. (B) Skin hyperextensibility on the neck. (C) Great toe was deviated laterally (equinovalgus) with medial protrusion of the metacarpophalangeal joint.
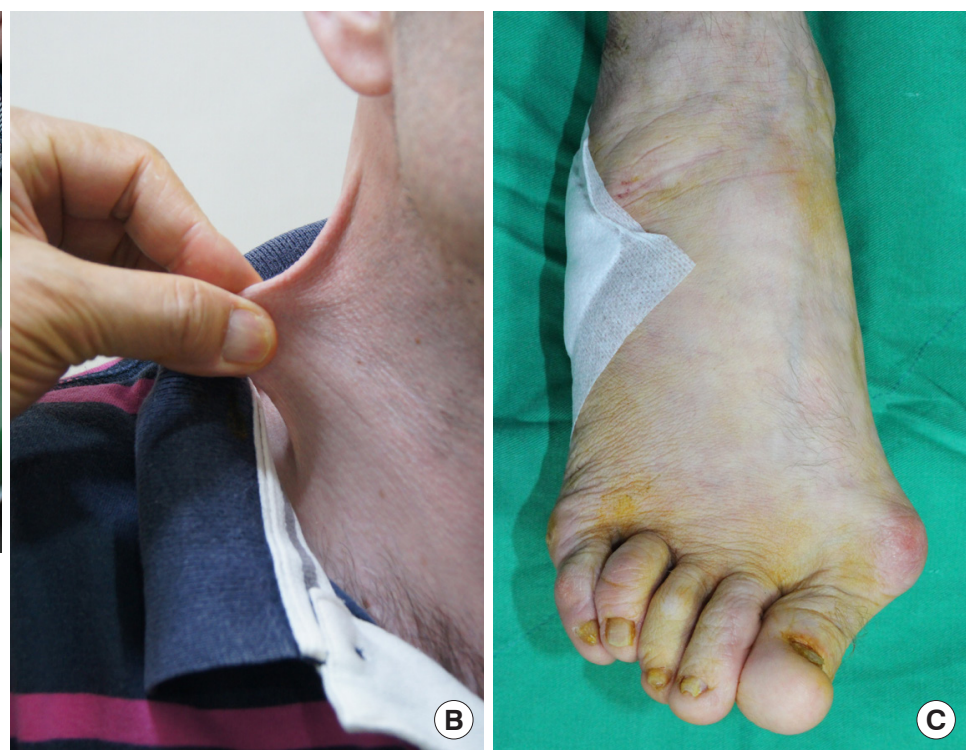
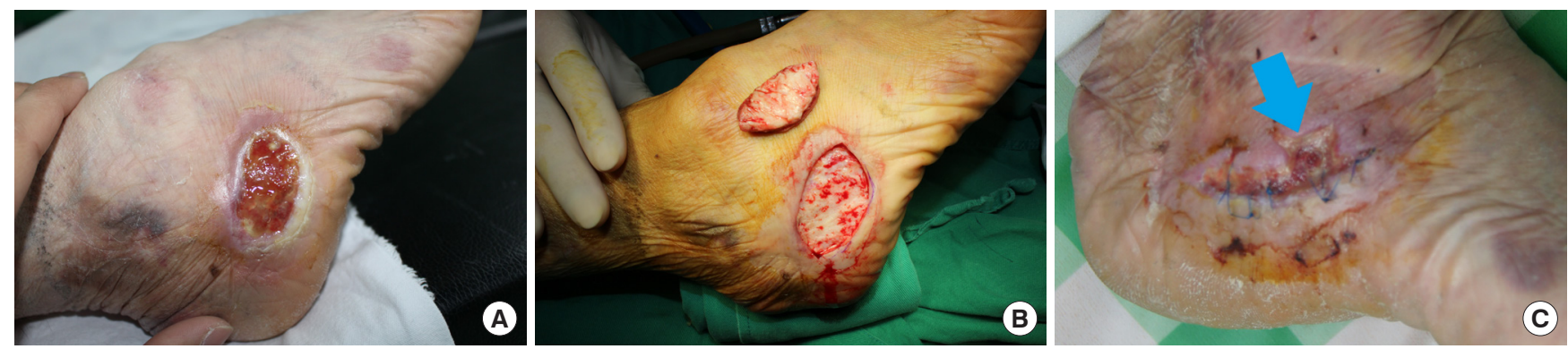

Fig. 2. Pre- and postoperative wound conditions. (A) When he visited our hospital a month after the injury, the open wound size became $5 \mathrm{~cm}$ horizontal length and 3-4 cm height, and the wound contained infected granulation tissue. (B) After wound treatment, the wound was elliptically excised suprafascially and wound margins were approximated after undermining. (C) Ten days after resuturing, partially necrotized wound, which was caused by bolster stitch, had not healed well on the anterior upper part (arrow).

and there were hypertrophic scars with callus formation on the top of protrusions (Fig. 1C). His open wound was initially a large hematoma formed after slipping down, and a part of the epidermal membrane covering the hematoma was removed from the private clinician for drainage of bloody fluid. Later, the dermatologist from the private clinic removed the dead skin on the wound. When he visited our hospital a month after the initial injury, his open wound was elliptically shaped with a $5-\mathrm{cm}$ horizontal length and a 3 to $4-\mathrm{cm}$ height. The wound had infected granulation tissue (Fig. 2A). After a 5-day of wound treatment, the wound improved. Elliptical excision of the wound and primary approximation were planned because of the skin hyperextensibility.

The open wound was elliptically excised on the suprafascial level (Fig. 2B) and the wound margins were subcutaneously underminded. Approximation of the wound margins was done with an interrupted 4-0 Vicryl subcutaneous sutures and 6-0 nylon skin sutures. Seven days after the operation, the wound had healed with a mild discharge from the posterior half of the wound. Three weeks after the operation, the wound was a little disrupted with a skin breakage from the nylon sutures. Resuturing was done after the debridement of wound margins, and two bolster stitches with 2-0 nylon were added on the skin about $1 \mathrm{~cm}$ apart from the suture line to keep wound margins tension free. However, two days after the bolster stitches, partial skin necrosis was developed on the one anterior suture area. Two bolster stitches were removed and wound dressing changes were repeated every day. However, partially necrotized wound had not healed well (Fig. 2C). The entire nylon sutures were taken out about 3 weeks after the reoperation because of the loosening of the nylon sutures.

During the wound treatment, the patient bought collagen wound dressing, FIBRACOL Plus (Acelity/Systagenix, San Antonio, TX, USA), from the UK and the wound began to heal at an accelerated rate after the application of FIBRACOL Plus (Fig. 3). After about 4 months after the first operation, the wound was well healed without aberrant scarring (Fig. 4), and he experiences no pain or discomfort while walking. 


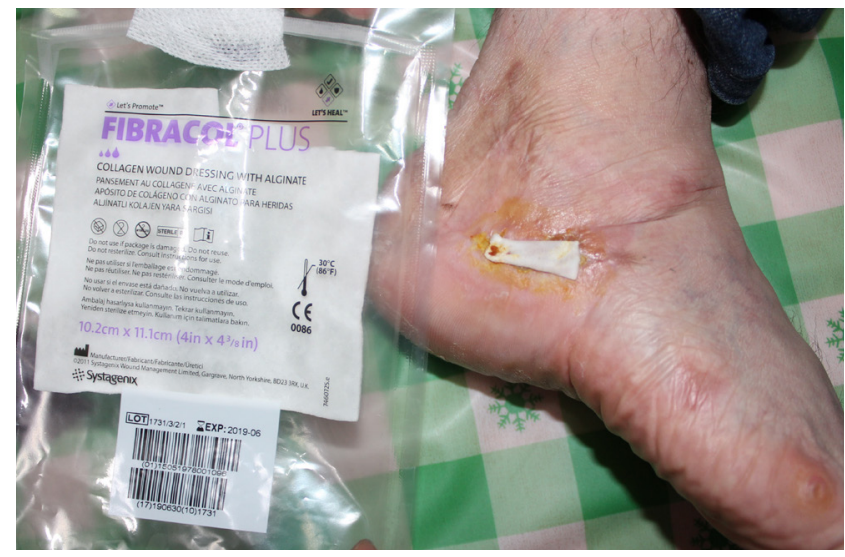

Fig. 3. Application of FIBRACOL Plus. FIBRACOL Plus is collagen wound dressing with alginate which was imported from the UK and it was changed once every 3 days.

\section{DISCUSSION}

In the six major types of EDS, classical, hypermobile, vascular, arthrochalasis types are inherited autosomal dominant but kyphoscoliotic and dermatosparaxis types are autosomal recessive. Proske et al. [3] described the characteristic clinical findings and election microscopic findings of the six major types of EDS in detail from their 20 years of experience.

The classical type is most common type and presented with hypermobile joints and soft, extremely fragile, hyperextensible skin displaying atrophic scarring. Pseudotumors develop at pressure points (elbows, knees) as well as the "knuckle" pads on hands and feet. Light microscopy in the architecture of dermal connective tissue is not specific, but the electron microscopic findings are characteristic in six major types. In the collagen bundles of the reticular dermis of the classical type, many ultrastructurally altered single fibrils with aberrant fibril contours, can be seen. The size and diameter of the fibril can vary greatly. The morphology of the elastic fibers is often highly inhomogenous.

The hypermobile type is characterized by an impressive hyperextensibility of large and small joints, often leading to the dislocation. The joint pathology often leads to swelling and arthritis. Joint pain is a characteristic symptom of the hypermobile type. Electron microscopy reveals single or multiple altered fibrils in the compact collagen bundles.

In the vascular type, the skin is thin and translucent, and is not hyperextensible but is commonly more tense. The tissue fragility leads to vulnerability of blood vessels, resulting in extensive bruising after a minimal trauma and wound healing is usually delayed. Life expectancy is markedly reduced due to vessel rupture with subsequent internal bleeding. Electron microscopy reveals remarkable variation in diameter of the colla-

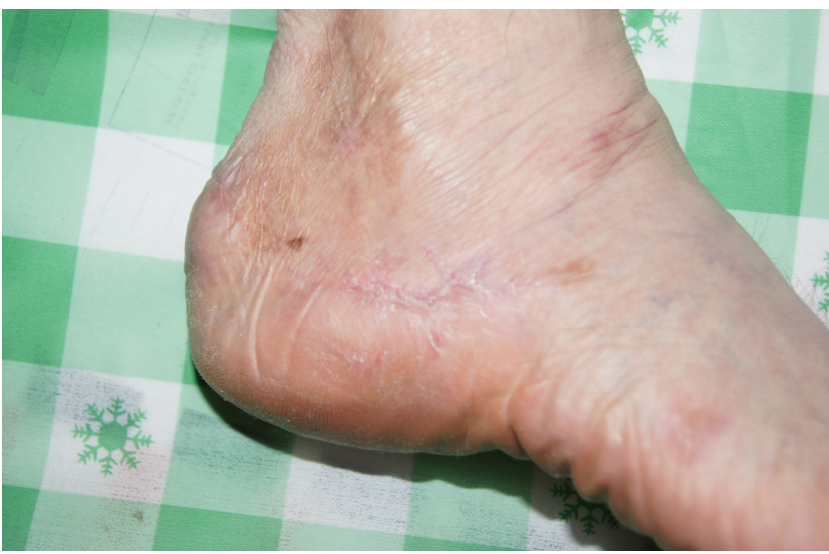

Fig. 4. Healed scar. The wound was well healed without aberrant scar about 4 months after first operation.

gen fibrils, loose packing of the bundles.

The main clinical feature of kyphoscoliotic type is the severe scoliosis, which may already be apparent at birth and the cause is muscular hypertonia and laxity of tendons. Cardinal symptoms are the ocular involvement such as retinal hemorrhage, retinal detachment and microcornea.

Characteristic findings of the arthrochalasis type are the prominent involvement of ligaments and joint capsules, including congenital bilateral hip dislocation. Characteristic of the dermatosparaxis type are blue sclera, lid edema, umbilical hernia, extreme skin fragility and typical electron microscopic finding is "hieroglyphic" collagen fibrils lead to diagnosis.

Surgical complications in EDS patients are comprised of mainly three parts [4]. Cutaneous complications include wound dehiscence, poor suture holding, and wide scar formation. Vascular complications include the friability of large vessels and woozing from small vessels. Gastrointestinal complications involve bowel anastomoses, which tend to dehisce during the first or the second postoperative week. Above complications can be summarized in two intraoperative problems: friable and fragile tissues that do not hold the suture material, and diffuse bleeding that is difficult to control. All surgical techniques should satisfy the basic principles of wound closure, which are provision of a tension-free repair and prevention of hematoma.

In the approximation of the wound margins with interrupted 4-0 Vicryl subcutaneous sutures and 6-0 skin nylon sutures in our case, two bolster sutures were applied to reduce the wound tension of the skin suture area but bolster stitch itself disturbed blood supply to the fragile tissue and necrotized the soft tissue. It took about 2 months to treat the necrotized tissue and the collagen wound dressing "FIBRACOL" was effective to treat the defect. 
In conclusions, recognition of EDS diagnosis in patients undergoing a repair of wide open wound affords the opportunity to anticipate poor wound healing, adjust the reconstructive plan, and therefore reduce the morbidity associated with the repair. When the wound is not too wide, one-stage closure is possible compared to non-EDS due to skin hyperextensibility, and it is advisable to remove skin suturing at least 5 days later than non-EDS cases. A mesh overlay graft to provide adequate musculofascial support is a good choice to reduce tension on the skin sutured area instead of bolster sutures. For the patients with EDS, the use of collagen wound dressing may be beneficial for accelerated wound healing.

\section{NOTES}

\section{Conflict of interest}

No potential conflict of interest relevant to this article was reported.

\section{Ethical approval}

The study was performed in accordance with the principles of the Declaration of Helsinki. Written informed consent was obtained.

\section{Patient consent}

The patient provided written informed consent for the publication and the use of his images.

\section{ORCID}

Bong Soo Baik https://orcid.org/0000-0002-8019-3310

Wu Seop Lee https://orcid.org/0000-0002-7540-4259

Ki Sung Park https://orcid.org/0000-0002-2672-2795

Wan Suk Yang https://orcid.org/0000-0002-2706-5678

So Young Ji https://orcid.org/0000-0002-6060-1452

\section{REFERENCES}

1. Byers PH, Murray ML. Heritable collagen disorders: the paradigm of the Ehlers-Danlos syndrome. J Invest Dermatol 2012; 132:E6-11.

2. Sharma NL, Mahajan VK, Gupta N, Ranjan N, Lath A. EhlersDanlos syndrome: vascular type (ecchymotic variant): cutaneous and dermatopathologic features. J Cutan Pathol 2009;36:486-92.

3. Proske S, Hartschuh W, Enk A, Hausser I. Ehlers-Danlos syndrome: 20 years experience with diagnosis and classification at the university skin clinic of Heidelber. J Dtsch Dermatol Ges 2006;4:308-18.

4. Girotto JA, Malaisrie SC, Bulkely G, Manson PN. Recurrent ventral herniation in Ehlers-Danlos syndrome. Plast Reconstr Surg 2000;106:1520-6. 